\title{
Partially disordered photonic- crystal thin films for enhanced and robust photovoltaics
}

\section{AUTHOR(S):}

Oskooi, Ardavan; Favuzzi, Pedro A.; Tanaka, Yoshinori; Shigeta, Hiroaki; Kawakami, Yoichi; Noda, Susumu

\section{CITATION:}

Oskooi, Ardavan ...[et al]. Partially disordered photonic-crystal thin films for enhanced and robust photovoltaics. APPLIED PHYSICS LETTERS 2012, 100(18): 181110.

\section{ISSUE DATE:}

2012-03

URL:

http://hdl.handle.net/2433/160629

\section{RIGHT:}

Copyright 2012 American Institute of Physics. This article may be downloaded for personal use only. Any other use requires prior permission of the author and the American Institute of Physics. The following article appeared in APPLIED PHYSICS LETTERS 100, 181110 (2012) and may be found at http://link.aip.org/link/?apl/100/181110 


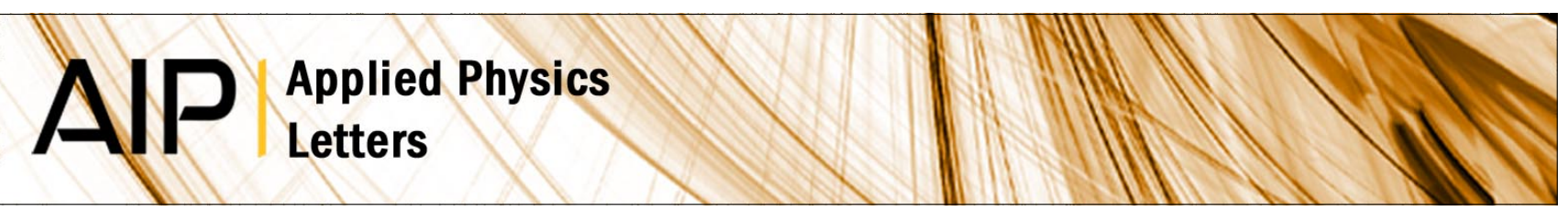

\section{Partially disordered photonic-crystal thin films for enhanced and robust photovoltaics}

Ardavan Oskooi, Pedro A. Favuzzi, Yoshinori Tanaka, Hiroaki Shigeta, Yoichi Kawakami et al.

Citation: Appl. Phys. Lett. 100, 181110 (2012); doi: 10.1063/1.4711144

View online: http://dx.doi.org/10.1063/1.4711144

View Table of Contents: http://apl.aip.org/resource/1/APPLAB/v100/i18

Published by the American Institute of Physics.

\section{Related Articles}

Raman spectroscopy of the interlayer shear mode in few-layer MoS2 flakes

Appl. Phys. Lett. 101, 101906 (2012)

Optical transport through finite superlattice modulated with three-component quasiperiodic defect

J. Appl. Phys. 112, 043524 (2012)

Generation of high-resolution kagome lattice structures using extreme ultraviolet interference lithography Appl. Phys. Lett. 101, 093104 (2012)

Back focal plane imaging spectroscopy of photonic crystals

Appl. Phys. Lett. 101, 081904 (2012)

Band structure of photonic crystal with dispersive and lossy materials using Dirichlet-to-Neumann wave vector eigen equation method

J. Appl. Phys. 112, 033112 (2012)

\section{Additional information on Appl. Phys. Lett.}

Journal Homepage: http://apl.aip.org/

Journal Information: http://apl.aip.org/about/about_the_journal

Top downloads: http://apl.aip.org/features/most_downloaded

Information for Authors: http://apl.aip.org/authors

\section{ADVERTISEMENT}

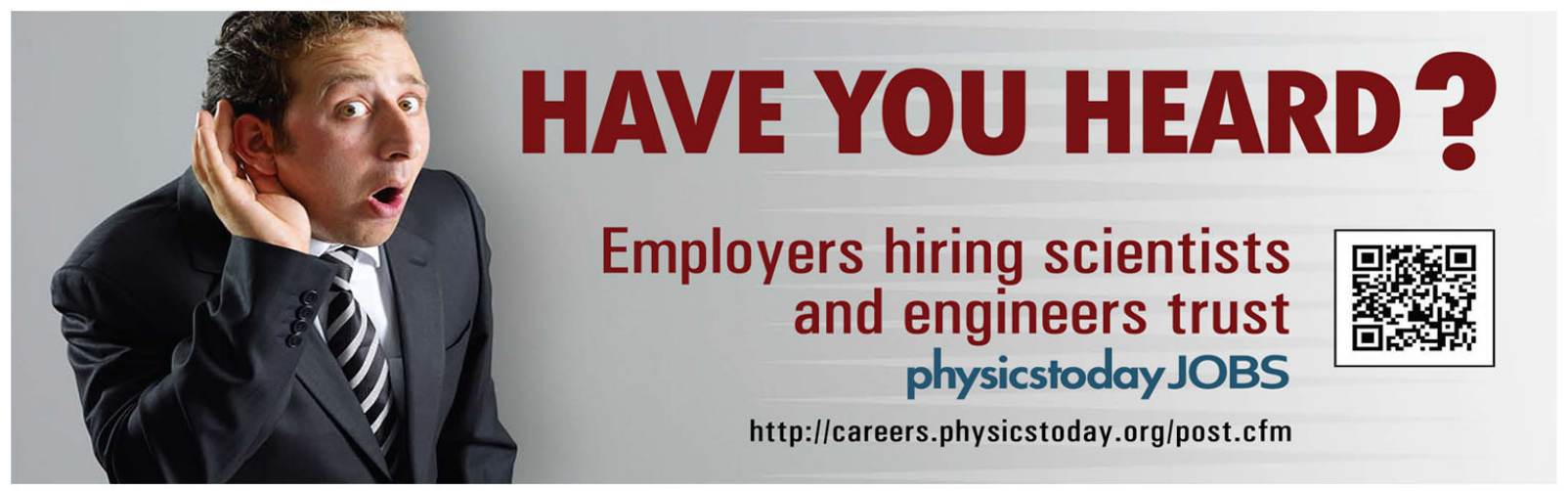




\title{
Partially disordered photonic-crystal thin films for enhanced and robust photovoltaics
}

\author{
Ardavan Oskooi, ${ }^{\text {a),b) }}$ Pedro A. Favuzzi, ${ }^{\text {b) }}$ Yoshinori Tanaka, Hiroaki Shigeta, \\ Yoichi Kawakami, and Susumu Noda \\ Department of Electronic Science \& Engineering, Kyoto University, Kyoto 615-8510, Japan
}

(Received 28 December 2011; accepted 18 April 2012; published online 3 May 2012)

\begin{abstract}
We present a general framework for the design of thin-film photovoltaics based on a partially disordered photonic crystal that has both enhanced absorption for light trapping and reduced sensitivity to the angle and polarization of incident radiation. The absorption characteristics of different lattice structures are investigated as an initial periodic structure is gradually perturbed. We find that an optimal amount of disorder controllably introduced into a multi-lattice photonic crystal causes the characteristic narrow-band, resonant peaks to be broadened resulting in a device with enhanced and robust performance ideal for typical operating conditions of photovoltaic applications. (C) 2012 American Institute of Physics. [http://dx.doi.org/10.1063/1.4711144]
\end{abstract}

Recent interest in enhancing the light-trapping functionality of thin-film photovoltaics has lead to a large number of proposals for different nanostructured designs that exploit the wave nature of incident light. ${ }^{1-7}$ The performance of these designs is typically optimized for only a limited set of operating conditions involving a reduced bandwidth, narrow-angular cone, and single polarization. In this letter, we show that by introducing a slight amount of disorder into a multi-lattice photonic-crystal (PC) slab, we can increase the light trapping over a wideband spectrum while also reducing its angular and polarization sensitivity. The presence of disorder itself may potentially make the design more feasible for large-scale production. We demonstrate that too much disorder, while improving the robustness of the design to properties of the incident light, actually lowers the light trapping relative to an optimum and we discuss the tradeoffs therein. The key design principle, we argue, is to controllably introduce just the right amount of disorder into the lattice so as to gradually broaden the sharp resonances of the PC; retaining aspects of the high-peak absorption while slightly reducing the dependence on specific-wave features.

The delicate interference effects that underlie waveoptics designs for photovoltaics often give rise to performance that is sensitive to the wave properties and thus makes it difficult to obtain enhanced light-trapping over a wideband spectrum spanning multiple angles and polarizations. A variety of different PC designs have already been proposed for improved light-trapping in thin-film photovoltaics. ${ }^{2-5}$ The core principle of such designs is a collection of narrow-band resonances $^{8-10}$ based on the coherent scattering of photons ${ }^{11}$ that together contribute to broadband absorption enhancement relative to an unpatterned slab of a given absorbing media (usually silicon or gallium arsenide). Figure 1 shows the absorption profile for two thin-film photovoltaic designs consisting of a $500 \mathrm{~nm}$-thick silicon ( $\mathrm{Si}$ ) absorbing layer, with and without a PC, having a metal back-reflector. The $\mathrm{PC}$ is formed by patterning a square lattice (periodicity

\footnotetext{
${ }^{a)}$ Electronic mail: oskooi@qoe.kuee.kyoto-u.ac.jp.

b) Ardavan Oskooi and Pedro A. Favuzzi contributed equally to this work.
}

$350 \mathrm{~nm}$ ) of oxide (refractive index 1.8) rods (radius $140 \mathrm{~nm}$, height $200 \mathrm{~nm}$ ) within the Si film (a cross section of the unit cell is shown in the schematic of Fig. 1). These specific design parameters for the PC, while not optimized for photon absorption over the given spectrum (quantified in the next section), represent a valid starting point for exploring the extent to which variations to the unperturbed lattice can improve light absorption. No front- or rear-surface texturing $^{12,13}$ is employed to boost scattering into the guided modes of the slab as we are only interested in the effect of the nanostructured lattice on the light trapping. A normally incident (later, we will consider a range of oblique angles) planewave spanning the wideband spectrum from $500 \mathrm{~nm}(\mathrm{Si}$ is strongly absorbing below $500 \mathrm{~nm}$ which renders less important the effect of the lattice to boost light trapping) to $1100 \mathrm{~nm}$ (the indirect bandgap of Si corresponds to a wavelength of $1107 \mathrm{~nm}$ ) impinges on the structure from above through a glass $(n=1.5)$ substrate. We use an open-source finite-difference time-domain (FDTD) simulation tool $^{14}$ to

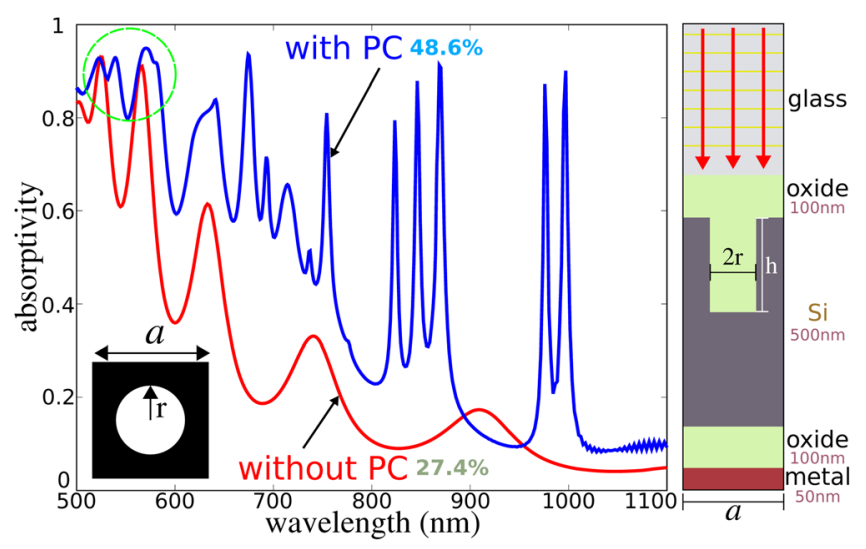

FIG. 1. Absorption versus wavelength profile at normal incidence for two thin-film photovoltaic designs: with a PC (blue) and without (bare slab, red). The PC consists of a square lattice (periodicity, $a=350 \mathrm{~nm}$ ) of circular rods $(r=140 \mathrm{~nm}, h=200 \mathrm{~nm})$ of oxide patterned in silicon (Si). The photonabsorption efficiency (from Eq. (1)) is a measure of light trapping relative to a perfect absorber. The peaks circled in green are in the limit of strong $\mathrm{Si}$ absorption and thus depend mostly on film thickness, not lattice design. 
compute the fractional absorptivity of the $\mathrm{Si}$ film. The absorption spectra for the PC show narrow, high-amplitude peaks-signatures of the coherent-resonant-Bloch modeswhereas the unpatterned slab has broad Fabry-Pérot resonances with low amplitude.

We can quantify the light-trapping efficiency of each design relative to an ideal perfect absorber (unity absorptivity over the wavelength interval of interest) by assuming that each absorbed photon with energy larger than the bandgap of crystalline Si generates an exciton which contributes directly to the short-circuit current. ${ }^{12}$ This corresponds to the following definition of photon-absorption efficiency:

$$
\frac{\int_{500 \mathrm{~nm}}^{1100 \mathrm{~nm}} \lambda \frac{d I}{d \lambda} A(\lambda) d \lambda}{\int_{500 \mathrm{~nm}}^{1100 \mathrm{~nm}} \lambda \frac{d I}{d \lambda} d \lambda},
$$

where $d I / d \lambda$ is the terrestrial power density per unit wavelength from a blackbody at a temperature of $5778 \mathrm{~K}$ (Ref. 15) and $A(\lambda)$ is the absorptivity computed from the simulations. Figure 1 shows that the introduction of a PC lattice into the absorbing Si layer results in a near doubling of the overall efficiency compared to an unpatterned slab ( $48.6 \%$ vs. $27.4 \%$ ). Somewhat non-intuitively, the effect of removing the absorbing material $(\mathrm{Si})$ to form the $\mathrm{PC}$ lattice has been to enhance the absorption via an increase in light localization. The sharp peaks of the PC (corresponding to a large $\mathrm{Q}$ factor ${ }^{11}$ ) indicate that coupling into (and out of) the modes is challenging and will depend strongly on the incident-wave conditions which we will verify later on. While a patterned design seems to be better for light trapping than an unpatterned one, what is less clear is whether the individual, narrow-band, large-amplitude peaks can be somehow broadened to further boost the overall absorption and the extent to which a given lattice design can be made robust with respect to different angles and polarizations of incident light.

The complex nature of the wave scattering that gives rise to the Bloch-mode resonances of the PC lattice depends delicately on perfect-structural order. It is natural to wonder then how the absorption spectra change as a gradual amount of disorder is introduced into the lattice. Figure 2 shows the evolution of the absorption profile for a steadily increasing amount of positional disorder. Each rod is perturbed from its position in the unperturbed lattice, a supercell consisting of $10 \times 10$ unit cells, by an amount $\Delta p$ chosen randomly from a uniform distribution of values between 0 and $\overline{\Delta p}$ for both orthogonal in-plane directions. Two separate simulations are made for each structure and the results averaged due to the random nature of the design. As the disorder progressively increases $(\overline{\Delta p}=0.05 a, 0.1 a, 0.2 a, 0.5 a)$, the peaks broaden more and more, converging to a profile resembling the unpatterned slab (Fig. 1, in red) but with higher absorption at each wavelength (peak broadening due to fabricationinduced disorder was first reported in a study by Chutinan and John ${ }^{16}$ but which did not investigate the effect of a controlled amount of disorder built into the design itself). In the limit of large disorder, no trace of the coherent scattering remains as the transition has been made to Anderson localization. ${ }^{17,18}$ Note that a transformation in the qualitative behavior of the absorption spectra occurs as the lattice changes from $\overline{\Delta p}=0.2 a$ (inset c) to $\overline{\Delta p}=0.5 a$ (inset d): in

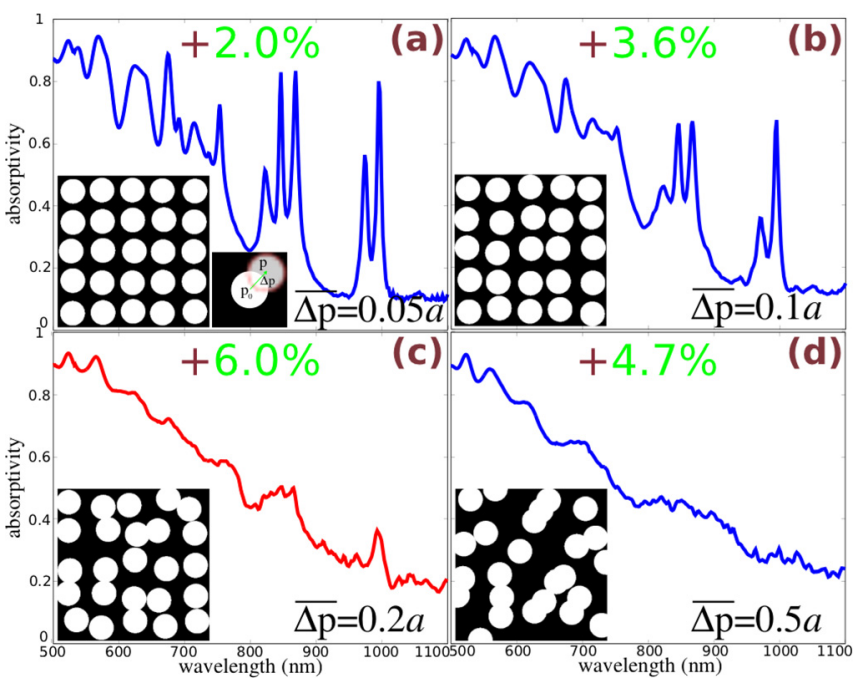

FIG. 2. The evolution of the absorption profile for a gradually increasing amount of positional disorder introduced into the PC. Each rod is perturbed from its position in the unperturbed $10 a \times 10 a$ lattice by an amount $\Delta p$ chosen randomly from a uniform distribution of values between 0 and $\overline{\Delta p}$. The efficiency values, in green, are the absolute change relative to the unperturbed lattice. Insets a, b, and c (in red, with maximal light trapping) show traces of the Bloch-mode resonances, while inset $\mathrm{d}$ is Anderson localized with sub-optimal absorption.

the former, traces of the resonant modes are still visible while in the latter they have completely disappeared. This suggests that, upon the addition of a relatively small amount of disorder, the wave behavior is still governed more or less by the scattering of Bloch-mode resonances while too much disorder results in a very different behavior marked by the incoherence of Anderson localization. ${ }^{19}$ Figure 2 also shows that a modest amount of disorder $(\overline{\Delta p}=0.2 a)$ maximizes the overall efficiency of the design (a $6.0 \%$ absolute increase beyond the unperturbed lattice) but that too much disorder $(\overline{\Delta p}>0.5 a)$ actually lowers the efficiency gains. A similar observation was previously made in the study of an inverseopal structure ${ }^{18}$ but the notion of a partially disordered PC has not yet been extended to light-trapping in thin films for photovoltaic applications. The effect of disorder on light trapping is twofold: (1) it can be used to tune the density of states (DOS), specifically the ratio of Bloch to Andersonlocalized modes and (2) it can be used to control the coupling rate into and out of the lattice. And hence, the result of little disorder is likely to increase the DOS slightly ${ }^{16}$ and also improve the coupling efficiency leading to an overall boost in the light trapping whereas large disorder eliminates the coherent modes entirely and enables the light to leak out as readily as it can couple in thus lowering the performance. A careful understanding of the role that disorder plays in these complimentary aspects of DOS and mode coupling may potentially leads to designs with better light-trapping behavior.

In addition to the large efficiency improvement of a partially disordered lattice compared to an unperturbed one, the slight broadening of the peaks offers another significant advantage: it reduces the dependence of the localized modes on the incident-wave properties. Up to this point, the absorption spectra have only been shown for the case of a normally incident planewave source. At off-normal incidence, the 


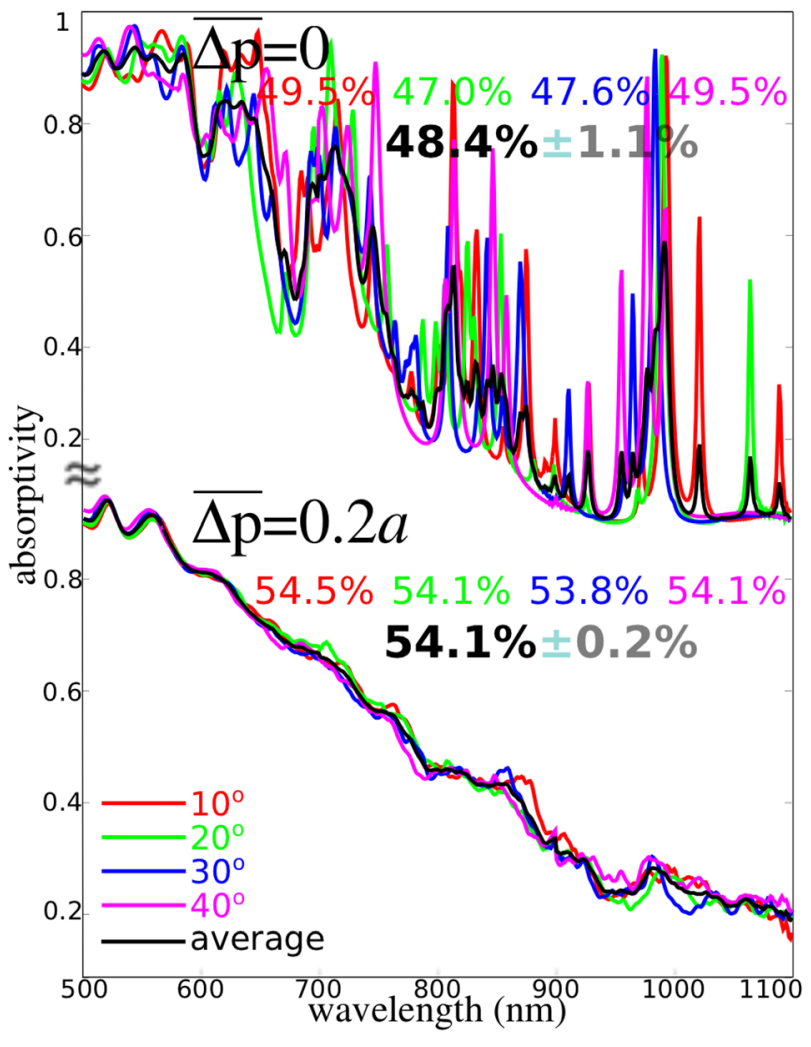

FIG. 3. Absorption profile for two different structures (the original unperturbed PC and one with optimal disorder) at four off-normal angles of incidence with the TE polarization. The absorption profile for the individual angles is colored, while the averaged profile is shown in black. Note large increase in robustness with disorder (i.e., standard deviation of the averaged efficiency decreases sharply). The TM polarization data (not shown) have similar characteristics.

polarization degeneracy is lifted enabling us to better investigate the dependence of a given design's efficiency on the wave-source conditions. Figure 3 shows the absorption profile for two structures-an unperturbed PC $(\overline{\Delta p}=0)$ and one with optimal disorder $(\overline{\Delta p}=0.2 a)$-at four different angles $\left(10^{\circ}, 20^{\circ}, 30^{\circ}\right.$, and $\left.40^{\circ}\right)$ for the TE polarization (electric field in the plane of incidence). Once again, we perform two simulations for the random structure and average the results to increase accuracy. As shown in the figure, the resonant peaks of the unperturbed PC have a strong dependence on the angle of the incident planewave. Moreover, there is a corresponding variation in the absorption efficiency from $49.5 \%$ to $47.0 \%$ which underscores just how sensitive the PC design's performance is to external factors (a lattice optimized for absorption at normal incidence showed an even larger range of variation from $53.1 \%$ to $47.3 \%$ ). The partially disordered optimal structure, on the other hand, shows much less sensitivity (quantified by a standard deviation value of the averaged efficiency that is a factor of five smaller) while a more disordered structure had even less sensitivity; yet, it is (expectedly) at the expense of a slightly lower overall efficiency (the trend is also true for the TM polarization). These results suggest that the choice of disorder strength requires a careful consideration of the trade off between enhancing the light-trapping on the one hand and making the structure more robust to angle and polarization on the other: too little disorder and the design performance depend sensitively on outside conditions, while too much disorder lowers the efficiency relative to an optimal amount (albeit any disordered lattice is superior to the unpatterned slab). Such an insight into the light-localization phenomenon of disordered structures points the way towards an interesting design strategy: find the maximum amount of disorder that still preserves some fundamental Bloch character of the modes and retains features of the large peak amplitudes to improve light trapping and robustness without broadening too much so as to be completely incoherent and leaky.

An important design consideration then, stemming from the observations made above, is to find a structure where the disorder (of different types and length scales) has both a small footprint and can be precisely controlled (similar to engineering disorder in surface-texturing approaches ${ }^{20}$ ) in order to better combine aspects of the high-absorption peaks of the unperturbed PC with the broadened, polarization- and angle-independent peaks of the strongly disordered lattice. To investigate this point in more detail, we now examine a multi-lattice design that consists of two sublattices, where the positional disorder of each sublattice can be independently tuned (the radius of all rods are equal). This multilattice structure, shown in Fig. 4, is an example of a lattice design that should offer a finer degree of control for introducing an optimal amount of disorder into the unperturbed structure to obtain the desired characteristics especially when compared to the single-lattice structure where all rods are perturbed in the same way. Figure 4 shows the absorption spectra, at normal incidence, of five different multi-lattice structures with steadily increasing disorder where only one of the two independent sublattices is perturbed. The broadening of the peaks happens gradually enough, acting to increase the absorptivity in regions where it was nearly negligible in the unperturbed lattice while simultaneously better preserving traces of the high-peak resonances, that the lighttrapping efficiency can be made to surpass that of the optimal single-lattice structure of Fig. 2 (in this example, the multi-lattice structure shows an efficiency gain of $7.7 \%$ at a

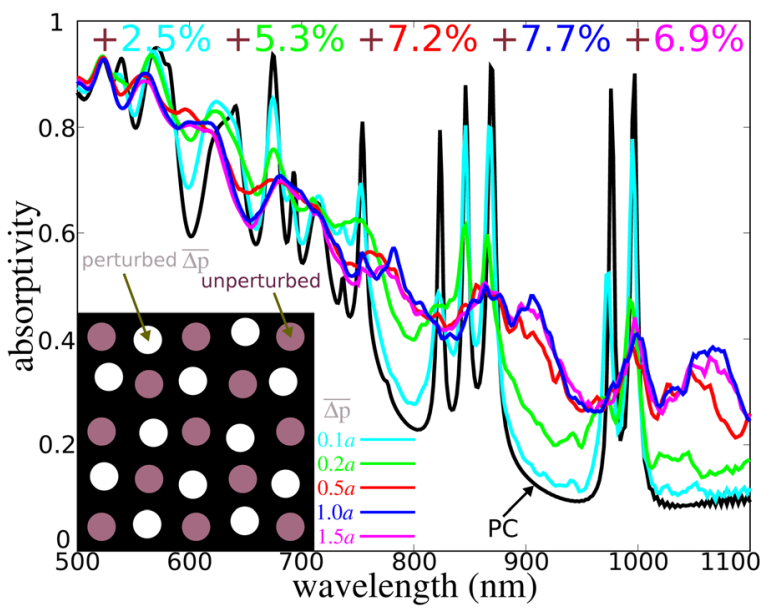

FIG. 4. Absorption profile, at normal incidence, for a multi-lattice structure composed of two independent sublattices (white and grey), where only one is perturbed $(\overline{\Delta p})$. Data for five different structures are shown (in color) with the unperturbed PC (in black) added for reference. Efficiency values for the five perturbed multi-lattice structures are the absolute change relative to the unperturbed lattice. 
$\overline{\Delta p}$ of $1.0 a$ ). (Note that since the disorder footprint is now smaller than before that more disorder is required to achieve similar gains and beyond.) By merging two sublattices into one structure this way, we are able to more effectively blend the large-peak-amplitude features of the coherent Bloch scattering with the broadening due to disorder.

While we demonstrated peak-broadening behavior using only positional disorder, the same phenomenon was observed for radial disorder although for a fixed supercell size the latter disorder type does not preserve the quantity of $\mathrm{Si}$ thus somewhat complicating the analysis. We also investigated the effect of disorder starting with an optimized unperturbed PC lattice $(53.2 \%$ at $a=500 \mathrm{~nm}$ and $r=150 \mathrm{~nm})$ and discovered similar trends of increasing and then decreasing absorption gains, although the maximum efficiency improvement was small by comparison (only $2.2 \%$ at a $\overline{\Delta p}$ of $0.05 a$ ). This might suggest that ultimately the optimal amount of disorder, different for each unperturbed starting lattice, will result in nearly identical maximum efficiencies (in this case around $55 \%$, likely limited by the material properties and film thickness of $\mathrm{Si}$ ) and that the main advantage of using a nonoptimal starting lattice is simply that more disorder can be incorporated therefore making the device more robust. Minor fluctuations in the absorption spectra, particularly in the longwavelength regime for large disorder, are strictly numerical noise due to the finite-supercell size and periodic boundary conditions. In fact, as the supercell size is increased (and with it the effect of randomness), these small oscillations diminish and the absorption profile becomes smoother. Another approach, more relevant for reducing noise in smallerwavelength features, would be to average the results over a larger number of random iterations (currently two). In addition to a uniform distribution for our random model, we also investigated a normal distribution where the mean positional disorder is fixed at 0 and the standard deviation is varied. The only significant difference, indeed a major one, in the absorption spectra between the two distribution models was that the uniform distribution leads to slightly less broadening due to their being an upper limit for the perturbation. It therefore appears that a uniform model of randomness is better at controlling the disorder than a normal one. A more comprehensive investigation of the role of different lattice designs and random distribution models will be undertaken in a future publication.

In summary, we have described a design principle for enhancing both light trapping and robustness using a par- tially disordered photonic-crystal multi-lattice structure where the amount of disorder, a parameter controlling the DOS as well as the coupling rate between external and internal modes, has both a small footprint and can be carefully chosen. As the performance of typical wave-optics based designs for photovoltaic applications depends sensitively on structural order and external conditions, this strategy may provide a route towards the realization of thin-film photovoltaics with high performance over a wide range of operating criteria.

This work was supported by Core Research for Evolutional Science and Technology (CREST) from the Japan Science and Technology Agency and the Japan Society for the Promotion of Science (JSPS).

${ }^{1}$ P. Bermel, C. Luo, L. Zeng, L. Kimerling, and J. Joannopoulos, Opt. Express 15, 16986-17000 (2007).

${ }^{2}$ D. Zhou and R. Biswas, J. Appl. Phys. 103, 093102 (2008).

${ }^{3}$ A. Chutinan, N. Kherani, and S. Zukotynski, Opt. Express 17, 8871-8878 (2009).

${ }^{4}$ Y. Park, E. Drouard, O. E. Daif, X. Letarte, P. Viktorovitch, A. Fave, A. Kaminski, M. Lemiti, and C. Seassal, Opt. Express 17, 14312-14321 (2009).

${ }^{5}$ S. Mallick, M. Agrawal, and P. Peumans, Opt. Express 18, 5691-5706 (2010).

${ }^{6}$ J. Zhu, Z. Yu, S. Fan, and Y. Cui, Mat. Sci. Eng. R. 70, 330-340 (2010).

${ }^{7}$ H. Atwater and A. Polman, Nature Mater. 9, 205-213 (2010).

${ }^{8}$ M. Imada, S. Noda, A. Chutinan, T. Tokuda, M. Murata, and G. Sasaki, Appl. Phys. Lett. 75, 316 (1999).

${ }^{9}$ M. Notomi, Phys. Rev. B 62, 10696-10705 (2000).

${ }^{10}$ S. Noda, M. Yokoyama, M. Imada, A. Chutinan, and M. Mochizuki, Science 293, 1123-1125 (2001).

${ }^{11}$ J. D. Joannopoulos, S. G. Johnson, R. D. Meade, and J. N. Winn, Photonic Crystals: Molding the Flow of Light, 2nd ed. (Princeton University Press, Princeton, NJ, 2008).

${ }^{12}$ M. Ghebrebrhan, P. Bermel, Y. Avniel, J. Joannopoulos, and S. Johnson, Opt. Express 17, 7505-7518 (2009).

${ }^{13}$ X. Sheng, S. Johnson, J. Michel, and L. Kimerling, Opt. Express 19, A841-A850 (2011).

${ }^{14}$ A. F. Oskooi, D. Roundy, M. Ibanescu, P. Bermel, J. D. Joannopoulos, and S. G. Johnson, Comput. Phys. Commun. 181, 687-702 (2010).

${ }^{15}$ ASTMG173-03, Standard Tables for Reference Solar Spectral Irradiances: Direct Normal and Hemispherical on 37 Degree Tilted Surface (ASTM International, West Conshohocken, PA, 2005).

${ }^{16}$ A. Chutinan and S. John, Phys. Rev. A 78, 023825 (2008).

${ }^{17}$ T. Schwartz, G. Bartal, S. Fishman, and M. Segev, Nature (London) 446, 52-55 (2007).

${ }^{18}$ C. Toninelli, E. Vekris, G. Ozin, S. John, and D. Wiersma, Phys. Rev. Lett. 101, 123901 (2008).

${ }^{19}$ M. Rechtsman, A. Szameit, F. Dreisow, M. Heinrich, R. Keil, S. Nolte, and M. Segev, Phys. Rev. Lett. 106, 193904 (2011).

${ }^{20}$ A. Chutinan, C. Li, N. Kherani, and S. Zukotynski, J. Phys. D: Appl. Phys 44, 262001 (2011). 